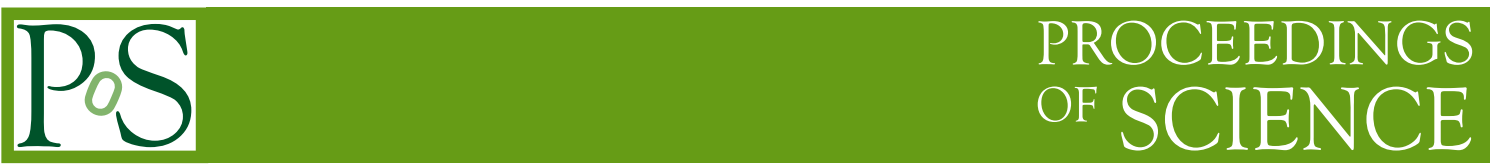

\title{
The search for rare decays at ATLAS and CMS
}

\author{
Riccardo Salvatico ${ }^{a, 1, *}$ \\ ${ }^{a}$ University of Kansas, \\ Lawrence KS 66045, USA \\ E-mail: riccardo.salvatico@cern.ch
}

The standard model describes a vast number of particles and their decays, including those of the $\mathrm{W}$ and $\mathrm{Z}$ bosons. Even though a large fraction of such decays have been known for a long time and the respective branching fractions have been measured with great accuracy, there remain several rare processes that have never been observed or even experimentally probed. Their observation could help validate the theoretical frameworks commonly used to calculate their branching fractions, but also provide viable alternatives to measure fundamental properties of the vector bosons, and possibly offer hints of new physics beyond the standard model. The two general-purpose experiments at the LHC cover a rich program of rare decay searches. This report focuses on those in the electroweak sector, including the search for $\mathrm{W}^{ \pm} \rightarrow \pi^{ \pm} \gamma$ and $\mathrm{W}^{ \pm} \rightarrow \pi^{ \pm} \pi^{ \pm} \pi^{\mp}$, performed by the CMS Collaboration, the measurement of the $\mathrm{Z} \rightarrow 4 \ell$ branching fraction, and the search for lepton flavor-violating decays $\mathrm{Z} \rightarrow \mathrm{e} / \mu \tau$, performed by the ATLAS Collaboration. Such searches and measurements exploit either partially or fully the integrated luminosity collected by the two experiments in the 2015-2018 data-taking period, and in some cases represent the state of the art in the respective fields.

The Ninth Annual Conference on Large Hadron Collider Physics - LHCP2021

7-12 June 2021

Online

${ }^{1}$ For the ATLAS and CMS Collaborations

* Speaker

(C) Copyright owned by the author(s) under the terms of the Creative Commons 


\section{Rare W boson decays}

Exclusive hadronic decays of the $\mathrm{W}$ boson are predicted by the standard model (SM), but their branching fractions $(\mathcal{B})$ are strongly suppressed. Large theoretical uncertainties generally affect their estimates: $\mathcal{B}(\mathrm{W} \rightarrow \pi \gamma)$ is expected in the range $10^{-9}-10^{-7}$ [1], whereas no calculation of $\mathcal{B}(\mathrm{W} \rightarrow \pi \pi \pi)$ is known to date. The observation of such decays would help to validate and improve the QCD factorization method, commonly used to perform these calculations. In addition, at future hadron colliders these decays might offer an alternative way to measure the $\mathrm{W}$ boson mass that is based solely on visible single-particle decay products.

The high energy thresholds for single photon triggers in 2016-2018 make an inclusive search for $\mathrm{W} \rightarrow \pi \gamma$ [2] unsuitable at CMS [3]. Therefore, a novel search strategy is adopted in Ref. [4], focusing on top quark-antiquark pairs $(\mathrm{t} \overline{\mathrm{t}})$. The muon or the electron emerging from one of the $\mathrm{W}$ bosons from $\mathrm{t}$ decay is used to select events at trigger level, whereas the rare decay topology, characterized by an isolated pion and a photon of large transverse momentum $\left(p_{\mathrm{T}}\right)$, is looked for from the other $\mathrm{W}$ boson. The presence of $\mathrm{b}$ quark jets helps to reduce the background from the hadronization of light-flavor quarks and gluons.

The analysis exploits the minimal amount of jet activity expected in the vicinity of the pion, which is produced via electroweak processes, to reject background pions. Thus, a pion-isolation variable is implemented, based on the sum of the $p_{\mathrm{T}}$ of all the particles contained in a cone around the pion itself. Such variable provides an outstanding signal discriminations against all the main background sources, including tti processes, Drell-Yan, and associated production of a W boson and a photon or jets.

Events are classified in a muon and an electron channel, depending on the flavor of the triggering lepton. A set of variables with good signal/background discriminating power, including the lepton, pion and photon momenta, the number of $b$ jets, and the pion isolation, is then used to train one boosted decision tree (BDT) per lepton channel. Upon the output of these BDTs, a signal- and a background-enriched regions of the pion-photon invariant mass $\left(m_{\pi \gamma}\right)$ are defined. The latter is fitted in the signal region (see Fig. 1) to measure $\mathcal{B}(\mathrm{W} \rightarrow \pi \gamma)$. No significant excess is observed above the expected background. An upper limit at $95 \%$ confidence level $\mathcal{B}(\mathrm{W} \rightarrow \pi \gamma)<1.50 \times 10^{-5}$ is set.

A different strategy is adopted in the search for $\mathrm{W} \rightarrow \pi \pi \pi$ [5], exploiting both at trigger and at reconstruction level an algorithm primarily designed for the reconstruction of $\tau$ hadronic decays $\left(\tau_{h}\right)[6,7] . \tau_{h}$ identification is seeded by energy deposits in the calorimeters, and then uses a combination of tracks in the silicon strip detector with charged particles in the jet. At trigger level, two $\tau_{h}$ candidates are required. Offline, the two pion candidates that match the trigger objects must be reconstructed in the 1-prong decay mode of $\tau_{h}$, and a third candidate with lower $p_{\mathrm{T}}$ requirements is searched for, still as a 1-prong $\tau_{h}$. The isolation of the pions is performed through discriminants implemented in the $\tau_{h}$ reconstruction algorithm, without making use of the vertex displacement information typically exploited in the identification of $\tau$ leptons.

A $40 \mathrm{GeV}$ lower threshold is set on the reconstructed $\mathrm{W}$ boson $p_{\mathrm{T}}$. This requirement provides a large efficiency for signal, while rejecting about two thirds of the background. The main contribution to the latter are QCD multijet events, fully estimated using data-driven methods. The normalization of other major background sources (Drell-Yan and $\bar{t} \overline{\text { }}$ is taken from simulation, whereas their 


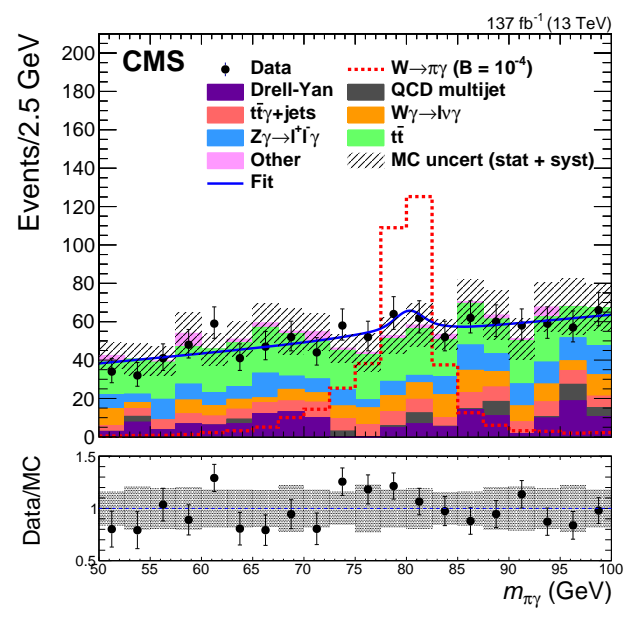

Figure 1: Event distribution as a function of $m_{\pi \gamma}$ for the combination of the lepton channels [4]. The simulated $\mathrm{MC}$ distribution for the signal is given by the dashed red line and corresponds to a $\mathcal{B}(\mathrm{W} \rightarrow \pi \gamma)=10^{-4}$. The blue line represents the best fit to the data.

distribution in the three-pion invariant mass variable is derived from studies in dedicated data control regions.

An upper limit $\mathcal{B}(\mathrm{W} \rightarrow \pi \pi \pi)<1.01 \times 10^{-6}$ is set, using an integrated luminosity corresponding to $77.3 \mathrm{fb}^{-1}$. The statistical uncertainty accounts for about $90 \%$ of the total uncertainty on this result, which is the first ever attempt to observe such decay.

\section{2. $\mathrm{Z} \rightarrow 4 \ell$ decay}

The work presented in Ref. [8] is a measurement of cross sections in final states with two pairs of opposite-sign, same-flavor leptons (either $4 \mu, 4 \mathrm{e}$, or $2 \mu 2 \mathrm{e}$ ) in pp collisions at $13 \mathrm{TeV}$ at ATLAS [9]. A number of SM processes can contribute to such final states, including quark-induced t-channel $q \bar{q} \rightarrow 4 \ell$, gluon-induced $g g \rightarrow 4 \ell$, Higgs boson-mediated s-channel production $\mathrm{H} \rightarrow Z^{*} Z^{*} \rightarrow 4 \ell$, and internal conversion in $\mathrm{Z}$ boson decays. The relative contribution of each of these processes to the total cross section depends on the 4-lepton invariant mass $m_{4 \ell}$ region considered.

The trigger selection requires the presence of one, two or three leptons. Then, a series of offline criteria define the so-called baseline leptons, including minimum $p_{\mathrm{T}}$ thresholds of the leading (20 $\mathrm{GeV})$ and sub-leading lepton $(10 \mathrm{GeV})$, a $\Delta R$ separation of the two leptons in a pair $>0.05$, and a dilepton invariant mass greater than $5 \mathrm{GeV}$ to suppress $\mathrm{J} / \psi$ decays. Leptons passing further isolation and impact parameter-based requirements are labeled as signal-like. Background events are primarily those containing a lepton quadruplet formed by a combination of prompt leptons from $\mathrm{Z} \gamma^{*}$ or $\mathrm{tt}$ processes, with additional leptons from hadron decay. The yield of such contributions is estimated using data regions enriched with non-prompt leptons. A fake factor is calculated as the ratio of the number of signal leptons to the number of baseline leptons that failed the signal selection, and is then applied to each baseline non-signal lepton in events where only the baseline selection is required.

The branching fraction $\mathcal{B}(\mathrm{Z} \rightarrow 4 \ell)=(4.41 \pm 0.30) \times 10^{-6}$ is extracted using the cross section measured in the $m_{4 \ell}$ region close to the $\mathrm{Z}$ mass, where the contribution of this process to the total 

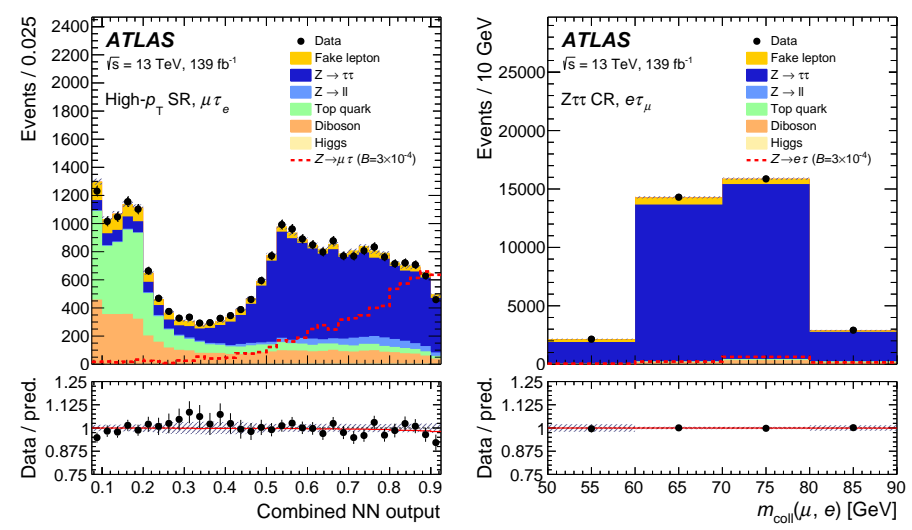

Figure 2: Observed and best-fit predicted distributions of the combined NN output in the SR (left), and of the lepton system invariant mass in the $\mathrm{CR} \mathrm{Z} \rightarrow \tau \tau$ (right) [11]. The expected signal is normalized to an arbitrary $\mathcal{B}(\mathrm{Z} \rightarrow \ell \tau)=3 \times 10^{-4}$. Lower panels: ratios of the observed yield (dots) and the best-fit background-plus-signal yield (solid line) to the best-fit background yield.

cross section is dominant. The estimate must be corrected for the contribution of sources other than $q \bar{q} \rightarrow 4 \ell$ and relies on simulation to estimate the fraction of $q \bar{q} \rightarrow 4 \ell$ events coming from single $\mathrm{Z}$ boson production, and that of events where no leptons originate from $\tau$ decays. This result is the most precise to date.

\section{Lepton-flavor-violating $\mathrm{Z}$ boson decays}

The large dataset collected at $13 \mathrm{TeV}$ by ATLAS in 2015-2018, corresponding to an integrated luminosity of $139 \mathrm{fb}^{-1}$, offers a vast sample of $\sim 8$ billion $\mathrm{Z}$ boson decays to be analyzed. The $\mathrm{Z} \rightarrow \mathrm{e} \tau$ and $\mathrm{Z} \rightarrow \mu \tau$ decays represent an interesting physics case, in that there exist no stringent indirect constraints to these processes, in contrast with the $\mathrm{Z} \rightarrow \mathrm{e} \mu$ case [10]. The observation of any of such lepton-flavor-violating processes would be an unambiguous indication of new physics.

The search in Ref. [11] considers only those events where the $\tau$ decays contain a lepton $\ell^{\prime} \neq \ell$, with $\ell$ the prompt lepton from the $\mathrm{Z}$ boson decay. The exclusion of events with same-flavor lepton pairs helps suppressing the $\mathrm{Z} \rightarrow \ell \ell$ background. A signal is expected to be characterized by two opposite-charge, back-to-back light leptons and a missing $p_{\mathrm{T}}$ that tends to be collinear with one of the charged leptons. Events containing $\tau_{h}$ decays are vetoed: these were analyzed in a previous search [12] and are thus used for combination.

Neural networks are trained to discriminate signal and background events, which are then classified in specific signal (SR) and control regions (CR). The branching fractions of the rare decays are extracted with a simultaneous fit to the neural network output in the SR (subdivided by lepton channel and by $p_{\mathrm{T}}$-range of the sub-leading lepton), the lepton system invariant mass $\left(m_{\text {coll }}\right)$ in the CR dominated by the $\mathrm{Z} \rightarrow \tau \tau$ background (see Fig. 2), and the event yield in the top quark-dominated CR. No significant excess is observed, and $95 \%$ confidence level upper limits are calculated for all the $\tau$ polarization scenarios. The combination with the $\tau_{h}$ results yields the most stringent limits to date for these processes, that is, for the unpolarized scenario $\mathcal{B}(\mathrm{Z} \rightarrow \mathrm{e} \tau)<5.0 \times 10^{-6}$ and $\mathcal{B}(\mathrm{Z} \rightarrow \mu \tau)<6.5 \times 10^{-6}$. 


\section{References}

[1] M. Mangano and T. Melia, Rare exclusive hadronic $W$ decays in a t $\bar{t}$ environment, EPJC 75 (2015) 258 [1410.7475].

[2] CDF Collaboration, Search for the rare radiative decay: $W \rightarrow \pi \gamma$ in p p collisions at $\sqrt{s}=1.96$ TeV, PRD 85 (2012) 032001 [1104 . 1585].

[3] CMS Collaboration, The CMS experiment at the CERN LHC, JINST 3 (2008) S08004.

[4] CMS Collaboration, Search for the rare decay of the $W$ boson into a pion and a photon in proton-proton collisions at $\sqrt{s}=13 \mathrm{TeV}$, PLB 819 (2021) 136409 [2011.06028].

[5] CMS Collaboration, Search for W boson decays to three charged pions, PRL 122 (2019) 151802 [1901.11201].

[6] CMS Collaboration, Reconstruction and identification of $\tau$ lepton decays to hadrons and $v_{\tau}$ at CMS, JINST 11 (2016) P01019 [1510.07488].

[7] CMS Collaboration, Performance of reconstruction and identification of $\tau$ leptons decaying to hadrons and $v_{\tau}$ in pp collisions at $\sqrt{s}=13 \mathrm{TeV}$, JINST 13 (2018) P10005 [1809.02816].

[8] ATLAS Collaboration, Measurements of differential cross-sections in four-lepton events in 13 TeV proton-proton collisions with the ATLAS detector, JHEP 07 (2021) 005 [2103. 01918].

[9] ATLAS Collaboration, The ATLAS experiment at the CERN Large Hadron Collider, JINST 3 (2008) S08003.

[10] S. Davidson, S. Lacroix and P. Verdier, LHC sensitivity to lepton flavour violating Z boson decays, JHEP 2012 (2012) 92 [1207. 4894].

[11] ATLAS Collaboration, Search for lepton-flavor-violation in Z-boson decays with $\tau$-leptons with the ATLAS detector [2105.12491].

[12] ATLAS Collaboration, Search for charged-lepton-flavour violation in Z-boson decays with the ATLAS detector, Nature Physics 17 (2021) [2010. 02566]. 